\title{
A 52-week, randomized, open-label study of aripiprazole versus blonanserin in the treatment of Japanese schizophrenia patients
}

\author{
Asuka Katsuki, Hikaru Hori, Kiyokazu Atake, Ryohei Igata, Yuki Konishi, Reiji Yoshimura
}

Department of Psychiatry, University of Occupational and Environmental Health, 1-1 Iseigaoka, Yahatanishi-ku, Kitakyushu, Fukuoka 807-8555, JAPAN

\begin{abstract}
Purpose: To evaluate the long-term efficacy and safety of aripiprazole and blonanserin in Japanese patients with schizophrenia.

Methods: In this 52-week, randomized, flexible-dose, open-label study, patients diagnosed with schizophrenia were randomized to receive aripiprazole $(n=14)$ or blonanserin $(n=12)$. The efficacy and safety of the drugs were evaluated using the Positive and Negative Syndrome Scale (PANSS) and the Clinical Global Impression-Severity Scale (CGI-S). The Drug-Induced Extrapyramidal Symptoms Scale (DIEPSS) was administered at baseline and 4, 12, 26, and 52 weeks after the initiation of treatment.
\end{abstract}

Results: Twelve patients (aripiprazole, $n=7$; blonanserin, $n=5$ ) completed this study. No significant differences in gender, episode, age, or PANSS total score were observed between the two groups at baseline. Both groups showed significant improvements during the study, with reductions in the total PANSS score (two-way repeated-measures ANOVA, both $\mathrm{p}<0.01$ ).

Discussion: In Japanese schizophrenia patients, aripiprazole and blonanserin showed comparable efficacy and tolerability during the 52-week study period. Both drugs showed good efficacy for treating schizophrenia, and the long-term therapeutic effect was maintained. Due to the high dropout rates, however, any conclusions must be considered preliminary and in need of replication.

(Clinical trial registration: UMIN000012729)

Keywords: schizophrenia, aripiprazole, blonanserin, randomized control trial, efficacy

Received September 8, 2017 / Accepted November 9, 2017 / Published December 15, 2017.

\section{Introduction}

Atypical antipsychotic drugs have been reported to be more efficacious and associated with a lower risk of extrapyramidal symptoms (EPS) and hyperprolactinemia than typical antipsychotics [1]. However, atypical antipsychotics have been reported to have higher metabolic risks, including weight gain, than typical antipsychotics [2]. Moreover, another meta-analysis revealed differences in safety, including metabolic risk and EPS, among atypical antipsychotics $[1,3,4]$. Thus, the rationale for selecting one drug over another, other than the patient's history of response, lack of response or side effects,

Corresponding Author: Asuka Katsuki, Department of Psychiatry, University of Occupational and Environmental Health, 1-1 Iseigaoka, Yahatanishi-ku, Kitakyushu, Fukuoka 807-8555, JAPAN, (Tel) +81936917253, (Fax) +81936924894, (E-mail) asuka-k@med.uoeh-u.ac.jp 
is currently limited. Because the effect size for efficacy is smaller than that for safety, several guidelines for the management of schizophrenia have recommended the use of the safer antipsychotic among the ones available for patients with schizophrenia [5-7].

The pharmacology of aripiprazole, developed in Japan, is unique; it exhibits partial agonist activity at dopamine D2/D3 receptors, which is associated with a low risk of hyperprolactinemia [8, 9], and partial agonist activity at 5-HT1A receptors and antagonist activity at 5-HT2A receptors. Additionally, aripiprazole carries a low risk of metabolic side effects such as weight gain, increased total cholesterol and blood pressure, hyperprolactinemia and sedation [3, 10]. Therefore, this drug has been widely recommended as a first-line treatment for schizophrenia $[11,12]$.

Blonanserin, which was also developed in Japan as a novel antipsychotic drug, has been approved for treating schizophrenia in Japan and Korea [13, 14]. In recent studies, the effect of blonanserin has been demonstrated to be equivalent to that of haloperidol and risperidone in primary endpoints and superior to haloperidol in improving negative symptoms in patients with schizophrenia $[15,16]$. This drug also carries a low risk of metabolic side effects and sedation [15].

Thus, both of these antipsychotic drugs were developed in Japan and are commonly used there. These antipsychotics have been reported to have a low incidence of sedation, weight gain, and cardiovascular risk and to sufficiently improve positive and negative symptoms. Accordingly, in a choice between 2 drugs with similar effects, hypotheses should be presented based on data from clinical studies with high evidence levels, such as randomized controlled trials. Recently, a 26-week study was conducted with 44 patients to compare the efficacy, tolerability, and safety of aripiprazole and blonanserin, and the results demonstrated equivalent efficacy and safety profiles for the 2 drugs [17]. However, headto-head and longer-term studies evaluating the safety and maintenance of antipsychotics in schizophrenia patients are lacking. Therefore, we performed a randomized controlled trial comparing blonanserin and aripiprazole for the clinical treatment of schizophrenia. The main objective of this study was to evaluate the effect of blonanserin versus aripiprazole on treating schizophrenia for 52 weeks.

\section{Methods}

\section{Participants}

This study enrolled patients (inpatients and outpatients) over 20 years of age who met the Diagnostic and Statistical Manual of Mental Disorders $4^{\text {th }}$ edition (DSM-IV) criteria for a primary diagnosis of schizophrenia, as established by the Structured Clinical Interview for the DSM-IV-TR (SCID-I/P). The patients were also required to have an illness duration of at least 6 months and no psychiatric hospitalizations in the 6 months prior to participating in the study.

The exclusion criteria included a DSM-IV-TR diagnosis other than schizophrenia, any medically significant abnormal electrocardiogram (ECG) results at screening, a history of substance abuse, neuroleptic malignant syndrome, diabetes, Parkinson's disease, an organic brain syndrome or acute medical condition, and significant suicide risk; additionally, pregnant women or women who had recently been breastfeeding ( $<1$ full cycle plus 1 week) were excluded. Subjects who were considered refractory to antipsychotic treatment based on their history, those who were responsive to clozapine treatment and those receiving long-acting antipsychotic treatment were also excluded.

Power analysis, with $\beta=0.20$ and $\alpha=0.05$, was conducted based on previous studies of the continuation rate for each antipsychotic. We had a projected sample size of approximately 100 patients (total of 200 patients), assuming a difference of $20 \%$.

This study is registered with the University Hospital Medical Information Network (UMIN) (No. UMIN000012729).

\section{Study design}

This randomized, flexible-dose, open-label, 52week clinical study was performed at the University of Occupational and Environmental Health from November 2012 to March 2016. The patients were randomized at a $1: 1$ ratio using the System for Automated Randomizations. Institutional review board approval was obtained from the University of Occupational and Environmental Health. All participants provided written informed consent. 
The study consisted of a screening phase and 2 treatment phases. Eligibility was determined during the screening phase (1-14 days). Following the screening period, the patients meeting the entry criteria were randomized at a $1: 1$ ratio. In Phase 1, from 0 to 12 weeks, subjects who were not already on aripiprazole or blonanserin monotherapy were cross-titrated during weekly visits from other antipsychotic(s) to oral aripiprazole or blonanserin monotherapy. The flexible dose ranges for blonanserin and aripiprazole were 4-24 mg/day and 3-30 $\mathrm{mg} /$ day, respectively. Maximal doses were based on the suggested optimal doses for schizophrenia patients in Japan (blonanserin, $24 \mathrm{mg} /$ day; aripiprazole, $30 \mathrm{mg} / \mathrm{day}$ ). The doses were adjusted according to the clinical judgments of the investigators. In Phase 2, from 13 to 52 weeks, the subjects received aripiprazole or blonanserin monotherapy.

Treatment compliance at each study visit was based on patient self-reports and calculated as the number of tablets taken divided by the number of tablets that should have been taken, multiplied by 100 .

Anticholinergic drugs were permitted for the treatment of EPS. Benzodiazepines were used as rescue medication for symptoms. Combined use of other psychotropic medications was prohibited.

\section{Clinical assessment}

Four certified psychiatrists (A.K, H.H, K.A and R.Y) who had at least 5 years of clinical experience in Japan evaluated the patients. To improve the inter-rater reliability, the training for each clinical evaluation was performed at the start of the study, and all raters participated in the training. The raters were not blinded about the group to which the patients belonged.

\section{Efficacy}

The primary outcome measure was the time to discontinuation for all reasons.

The secondary efficacy assessments were the mean change in the Positive and Negative Syndrome Scale (PANSS) total score from baseline to endpoint (week 52), the onset of effects (the first PANSS assessment that showed a significant difference in the PANSS total score between aripiprazole and blonanserin that was maintained for the remainder of the study) and the change from baseline to endpoint in the PANSS subscale scores and Clinical Global Impression-Severity Scale (CGI-S) scores.

\section{DIEPSS}

The Drug-Induced Extrapyramidal Symptom Scale (DIEPSS) [18] was used to assess EPS at baseline and $4,12,26$, and 52 weeks.

\section{Statistical analysis}

A randomized sample of patients who received at least one dose of the study medication formed the intention-to-treat population. We used Kaplan-Meier survival curves to estimate the time to discontinuation of treatment. The treatment groups were compared using Cox proportional hazards regression models.

The statistical analysis included a two-way repeated-measures analysis of variance (ANOVA) based on the estimated marginal means by fitting a linear mixed model. The linear mixed model that we used included the two treatment arm effects (aripiprazole vs. blonanserin) and time (baseline and weeks 4, 12, 26, and 52). This linear mixed model was used to assess the effects of the intervention on the primary outcome with treatment (aripiprazole/blonanserin) at 52 weeks. This model was fitted with the treatment (aripiprazole/blonanserin) as the fixed effect and the PANSS subscale, PANSS total, CGI-S, or DIEPSS scores as the dependent variable with no random effects. Moreover, pairwise and non-pairwise comparisons based on the estimated marginal means were used with Bonferroni correction for multiple comparisons, and within-group comparisons based on the estimated marginal means were used with Bonferroni correction for multiple comparisons.

\section{Results}

\section{Patients, treatment, and time to discontinuation}

A total of 26 patients were randomly assigned to receive either blonanserin $(n=12)$ or aripiprazole $(n=14)$. All patients were included in the final analysis (Figure 1). The demographic and clinical characteristics at baseline were similar in the two groups (Table 1). Seven patients $(50 \%)$ in the blonanserin group and $7(58.3 \%)$ in the aripiprazole group discontinued their treatment due to adverse effects (AEs). Overall, the proportions of patients who completed the assigned treatments were similar in the blonanserin $(\mathrm{n}=5 ; 41.7 \%)$ and aripiprazole $(\mathrm{n}=7 ; 50 \%)$ groups (Figure 1). Among the completers, the mean daily doses upon study completion were $10.7 \pm 4.6 \mathrm{mg}$ for blonanserin and $19.2 \pm$ $7.8 \mathrm{mg}$ for aripiprazole. Benzodiazepine doses did 


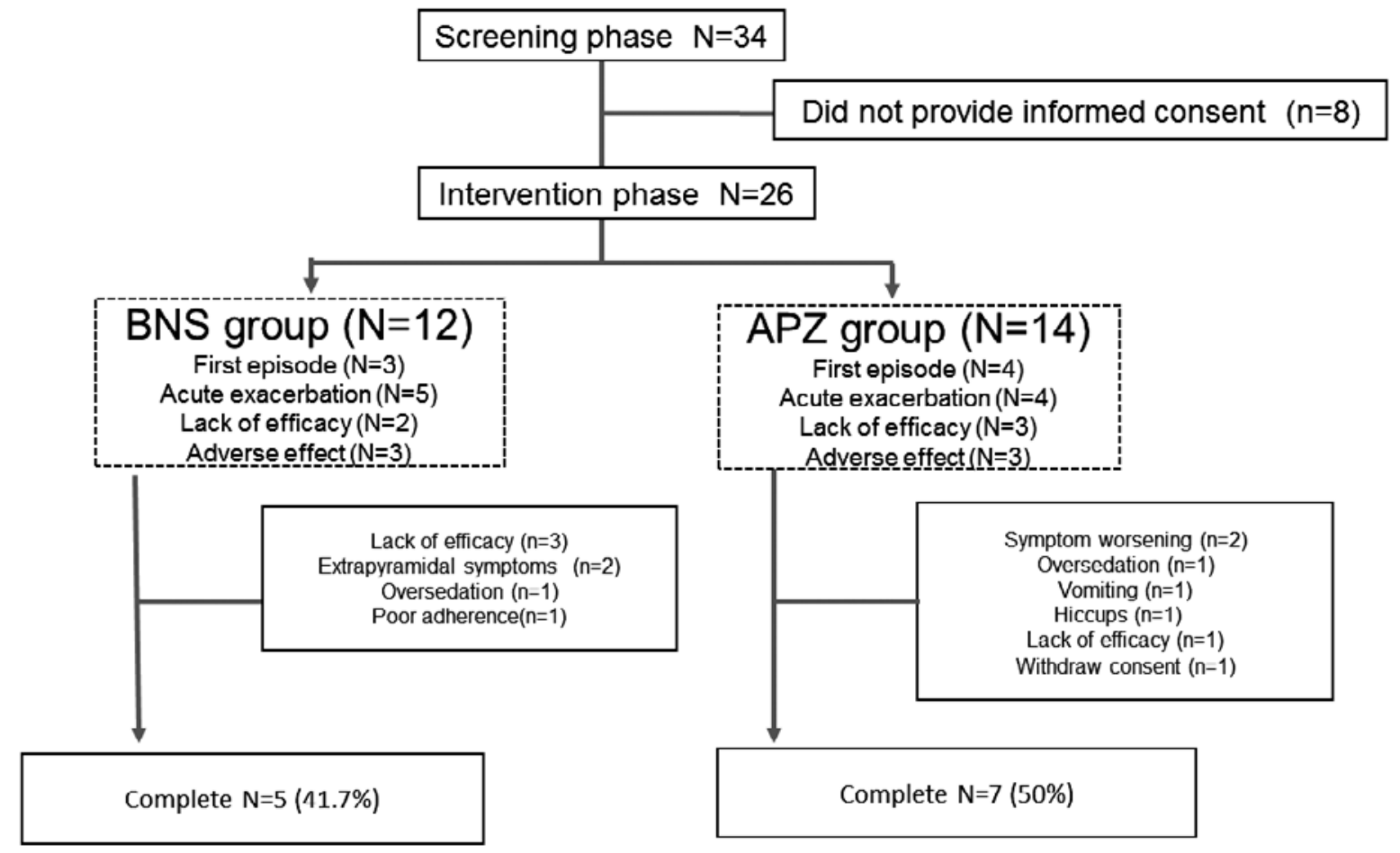

Figure 1. Subject flowchart of analysis

Table 1. Demographic data

\begin{tabular}{lccc}
\hline & APZ $(\mathrm{n}=14)$ & BNS $(\mathrm{n}=12)$ & $\mathrm{p}$-value \\
\hline Sex (M/F) & $7 / 7$ & $7 / 5$ & $0.713 \mathrm{a}$ \\
Hospitalization (In/Out) & $2 / 12$ & $4 / 8$ & $0.365 \mathrm{a}$ \\
Marital status (Yes/No) & $3 / 11$ & $3 / 9$ & $>0.99 \mathrm{a}$ \\
Unemployed (\%) & 57.1 & 83.3 & $0.216 \mathrm{a}$ \\
Mean \pm SD & & & \\
Age & $38.4 \pm 8.9$ & $40.2 \pm 15.4$ & $0.723 \mathrm{~b}$ \\
Education (years) & $12.8 \pm 2.3$ & $12.6 \pm 1.6$ & $0.764 \mathrm{~b}$ \\
Antipsychotic dose & $107.5 \pm 107.5$ & $195.8 \pm 273.4$ & $0.311 \mathrm{~b}$ \\
(mg/day; chlorpromazine equivalent) & & & \\
PANSS_0W-P & $23.6 \pm 4.8$ & $28.6 \pm 6.9$ & $0.026 \mathrm{~b}$ \\
PANSS_0W-N & $23.6 \pm 3.6$ & $22.6 \pm 4.0$ & $0.516 \mathrm{~b}$ \\
PANSS_0W-G & $45.9 \pm 5.5$ & $51.1 \pm 9.8$ & $0.099 \mathrm{~b}$ \\
PANSS_0W-T & $92.9 \pm 9.4$ & $101.8 \pm 16.0$ & $0.088 \mathrm{~b}$ \\
CGI-0W & $4.0 \pm 0.8$ & $4.6 \pm 0.8$ & $0.072 \mathrm{~b}$ \\
DIEPSS_0W & $0.7 \pm 1.5$ & $0.7 \pm 1.2$ & $0.932 \mathrm{~b}$ \\
\hline
\end{tabular}

a: Fisher's exact test, b: unpaired t-test

not differ significantly between the two groups (Table 2).

The results revealed no significant difference in the mean time to discontinuation between the aripiprazole and blonanserin treatment groups (98.0 [27.9168.1] days vs. 307.0 [123.8-490.2] days; HR= 0.981 [0.343-2.803], p=0.971) (Figure 2, Table 3).

\section{Efficacy results}

The linear mixed model for repeated-measures ANOVA indicated that the blonanserin group showed a significant change in positive symptom scores $(\mathrm{F}=3.286, \quad \mathrm{p}=0.019)$. Blonanserin and aripiprazole treatments did not show significant differences in other outcome assessments (PANSS negative, general, total scale, general psychopathology scale, and CGI scores) (Table 4). 
Table 2. Diazepam equivalent dosage during the study

\begin{tabular}{|c|c|c|c|c|c|c|}
\hline & & $0 \mathrm{w}$ & $4 \mathrm{w}$ & $12 \mathrm{w}$ & $26 \mathrm{w}$ & $52 \mathrm{w}$ \\
\hline \multicolumn{7}{|c|}{ diazepam equivalent dosage ( $\mathrm{mg} /$ day) } \\
\hline APZ & & $4.9 \pm 6.5$ & $5.6 \pm 6.4$ & $6.0 \pm 6.6$ & $6.0 \pm 6.6$ & $5.2 \pm 6.4$ \\
\hline BNS & & $2.0 \pm 2.7$ & $2.0 \pm 2.7$ & $2.0 \pm 2.7$ & $2.0 \pm 2.7$ & $2.8 \pm 4.2$ \\
\hline & P-value (APZ vs. BNS) & $0.374 \mathrm{a}$ & $0.273 \mathrm{a}$ & $0.232 \mathrm{a}$ & $0.232 \mathrm{a}$ & $0.476 \mathrm{a}$ \\
\hline
\end{tabular}

a two-way repeated-measured ANOVA

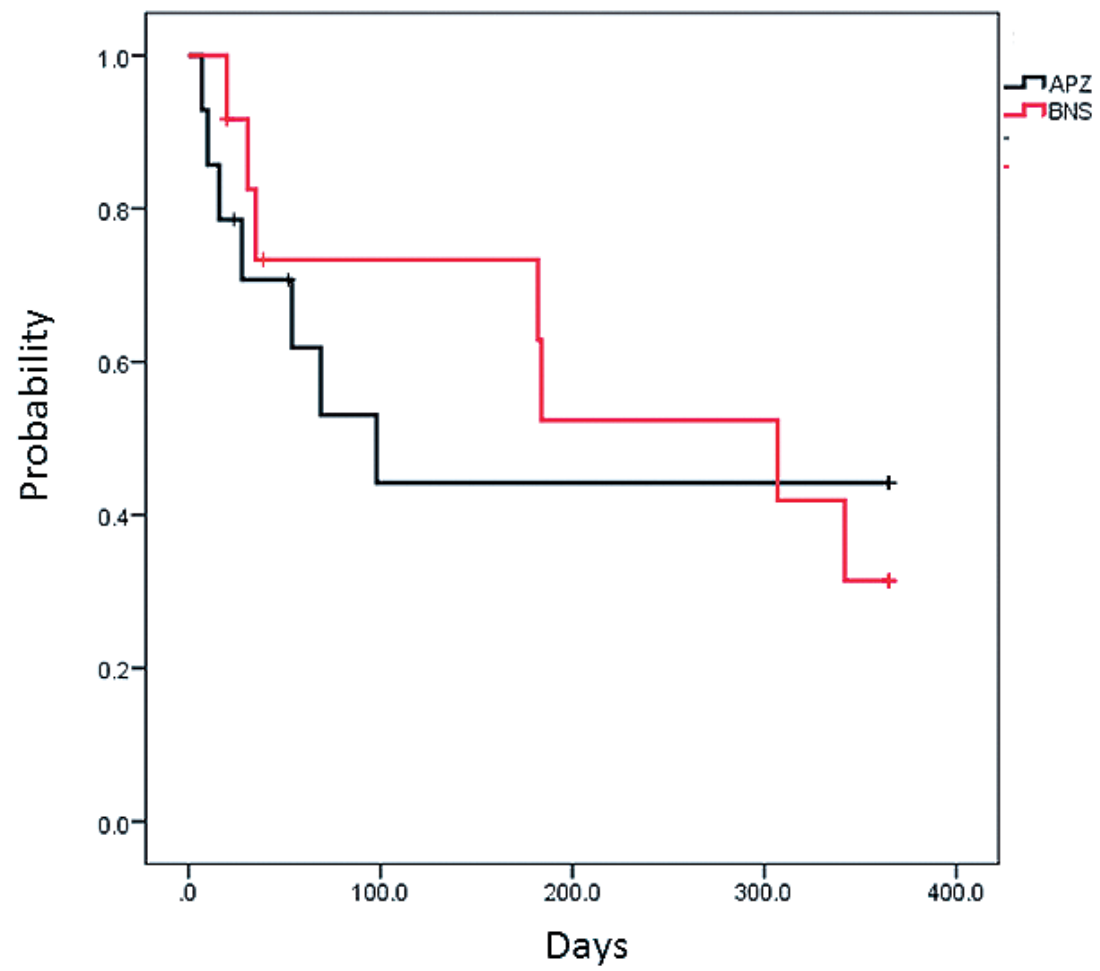

Figure 2. Kaplan-Meier estimate of time to discontinuation for any reason during the observation period

The within-group comparison for both groups demonstrated robust improvement in the PANSS total score from 4 weeks. The change in the PANSS positive score relative to the baseline score in weeks $4,12,26$ and 52 indicated a significant improvement for the blonanserin group; however, the aripiprazole group showed no significant improvement in assessment time from baseline. Neither group demonstrated a significant improvement in the PANSS negative score from baseline to endpoint. The change in the PANSS general score relative to the baseline score in weeks 4, 12, 26 and 52 represented a significant improvement for the blonanserin group, and in weeks 12, 26 and 52, the aripiprazole group showed a significant improvement. However, only the blonanserin group showed a significant change from baseline to endpoint in the CGI score (Table 4).
The between-groups comparison revealed significant differences in the PANSS positive score and the CGI at baseline. In the other assessments and evaluation times, no significant differences were observed (Table 2).

The discontinuation rate during the study was $50 \%$ in the aripiprazole treatment group and $41.7 \%$ in the blonanserin treatment group (Figure 1). The main reasons for discontinuation included lack of efficacy ( $\mathrm{n}=4$ for aripiprazole and $\mathrm{n}=2$ for blonanserin) and AEs ( $n=3$ for aripiprazole and $n=3$ for blonanserin) (Figure 1).

\section{AEs}

The rates of AEs and side effects are presented in Figure 1. During double-blind treatment, the most common treatment-emergent AEs that occurred more frequently in blonanserin-treated than in 
Table 3. Difference in the mean time to discontinuation between the aripiprazole and blonanserin treatment groups

\begin{tabular}{lcc}
\hline & APZ $(\mathrm{n}=14)$ & BNS $(\mathrm{n}=12)$ \\
\hline Discontinuation of treatment for any cause & & \\
Discontinuation, $\mathrm{n}(\%)$ & $7,50.0$ & $7,58.3$ \\
Kaplan-Meier time to discontinuation, days & & \\
Median [95\% CI] & $98.0[27.9,168.1]$ & $307.0[123.8,490.2]$ \\
Cox-model treatment comparison & & \\
APZ & & $0.981[0.343,2.803]$ \\
Hazard ratio [95\% CI] & 0.971 \\
P-value & \\
\hline
\end{tabular}

aripiprazole-treated subjects were EPS, whereas the most common treatment-emergent AEs that occurred more frequently in aripiprazole-treated subjects than in blonanserin-treated subjects were symptom worsening, vomiting, and hiccups.

The only serious AE reported by $>1 \%$ of patients in either group was psychotic disorder. The overall number of discontinuations due to treatmentemergent AEs was low. During double-blind treatment, $58.3 \%(\mathrm{n}=7 / 12)$ of the blonanserin patients and $50 \%(n=7 / 14)$ of the aripiprazole patients discontinued use due to treatment-emergent AEs. No death or suicide-related AEs were reported during the study.

AE assessments with the DIEPSS did not show significant differences in the changes for either group from baseline to week 52 using the linear mixed model $(\mathrm{F}=0.484, \mathrm{p}=0.748)$.

\section{Discussion}

This paper is the first randomized controlled trial of aripiprazole vs. blonanserin in schizophrenia patients over a 52-week study period. A previous randomized controlled study reported similar efficacy and safety profiles for aripiprazole and blonanserin in schizophrenia patients over a 26 -week study period [17]. We observed patients for up to 52 weeks and found no significant differences in the mean time to discontinuation between the aripiprazole and blonanserin treatment groups. The PANSS scores of the two antipsychotics improved significantly from the baseline scores. Our results show that both antipsychotics can improve schizophrenia symptoms and have equivalent efficacy and tolerability. In the within-group comparison, only the blonanserin group showed a significant change from baseline to endpoint in the CGI and PANSS positive scores. This result could be due to the baseline differences between the two groups. We found no significant differences in the total, negative and general PANSS scores; however, the blonanserin group showed a significant change in the positive symptom score based on the linear mixed model. Thus, blonanserin may reduce positive symptoms to a greater extent than aripiprazole.

Regarding the long-term use of antipsychotics, consideration should be given to AEs such as weight gain, metabolic side effects, and tardive dyskinesia. Akathisia was the most commonly reported EPSrelated $\mathrm{AE}$ in the aripiprazole group, whereas parkinsonism was the most common reported EPSrelated $\mathrm{AE}$ in the blonanserin group. Both groups demonstrated equivalent safety.

One of the major limitations of this study was its open-label design. The open-label design can generate bias, as both the patient and the physician are aware of which groups are receiving what type of treatment. A second limitation was that we did not evaluate patients for anxiety and depression by using scales such as the Montgomery Asberg Depression Rating Scale or the Hamilton Rating Scale for Anxiety $[19,20]$. A third limitation was related to the method of switching from the antipsychotics in administration before this study to aripiprazole or blonanserin, as the current antipsychotics were interrupted before the start of the study. Some reviews suggest that the dose should gradually be decreased when switching to aripiprazole [21, 22]. By contrast, a recently systematic review and metaanalysis found no significant differences in any clinical outcomes between the 2 approaches of examining immediate and gradual antipsychotic discontinuation in antipsychotic switching [23]. Therefore, the abrupt termination of the antipsychotics might have affected the study results. However, we 


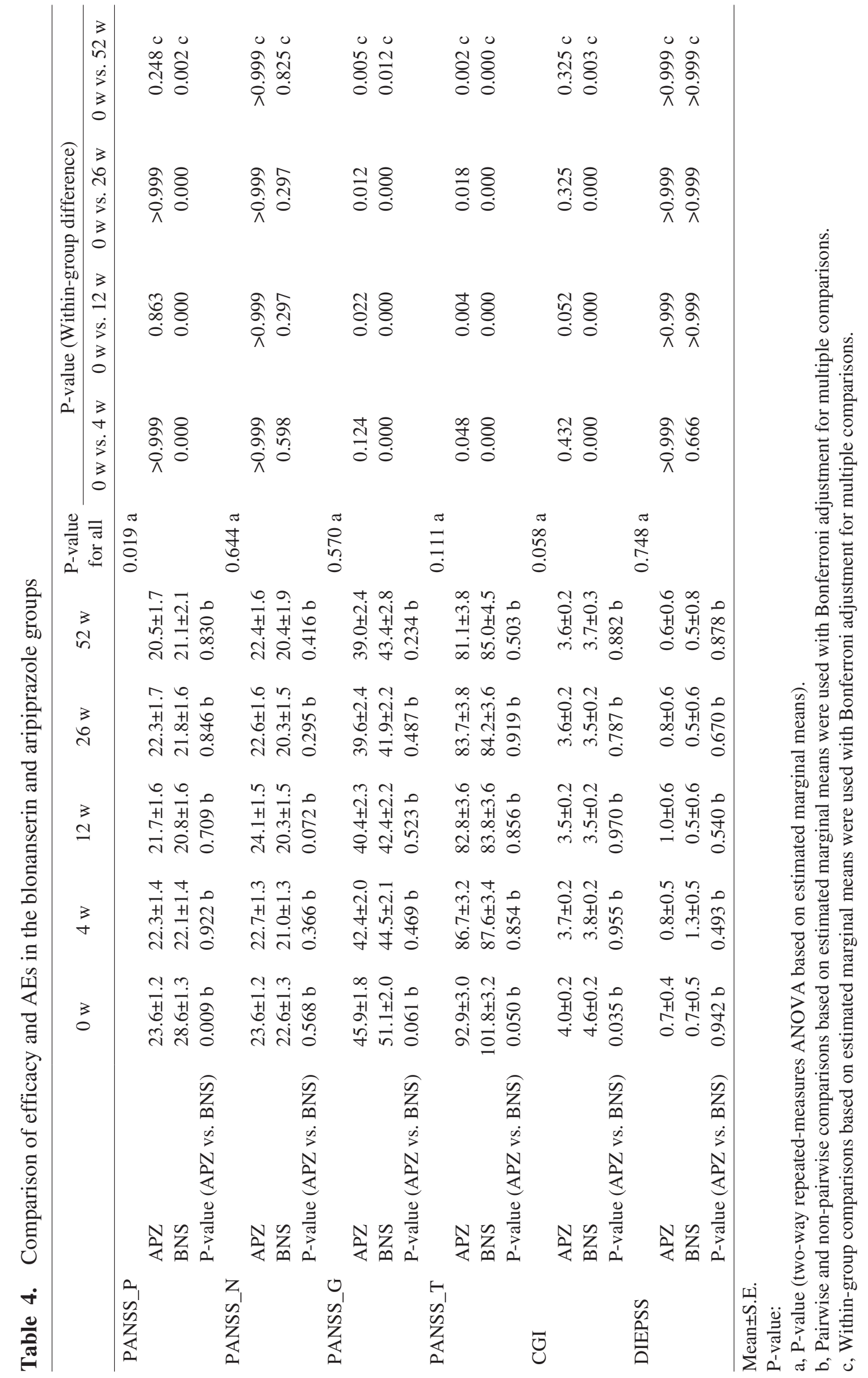


received no reports about the methods for switching to blonanserin. The fourth limitation is the small sample size of this study. Considering the result of the power test, Type II errors might occur. We did not perform regression analyses of the effect of the baseline scores on the PANSS and CGI in the two groups because our sample was small. On the other hand, there were no withdrawals of consent in either group. A fifth limitation is a lack of assessment of functioning, cognition and subjective perspectives (e.g., quality of life), and assessment of tolerability was confined to extrapyramidal symptoms in this study.

In conclusion, our findings suggest that aripiprazole and blonanserin are both effective and well tolerated in schizophrenia patients. However, additional adequately well-designed, double-blind, randomized controlled studies are needed to further compare the efficacy and safety of aripiprazole and blonanserin.

\section{Conflict of interest}

Dr. Hori has received speaker's honoraria from Dainippon Sumitomo, Eli Lilly, Janssen, Otsuka, Shionogi, Meiji and Pfizer.

Dr. Katsuki has received speaker's honoraria from Dainippon Sumitomo, Meiji.

Dr. Atake has received speaker's honoraria from Dainippon Sumitomo and Eli Lilly.

Dr. Igata and Dr. Konishi declare that no conflicts of interest are associated with this manuscript.

Dr. Yoshimura has received speaker's honoraria from Dainippon Sumitomo Eli Lilly, Janssen, Otsuka, Shionogi, Meiji, Pfizer, Novartis and Mochida.

\section{ACKNOWLEDGEMENT}

Dr. Kenji Yamada and Yuki Tokutsu helped to recruit the patients.

Asuka Katsuki, Hikaru Hori. contributed equally to this work.

\section{REFERENCES}

[1] Leucht S, Corves C, Arbter D, et al. Secondgeneration versus first-generation antipsychotic drugs for schizophrenia: A meta-analysis. Lancet 2009; 373: 31-41.

[2] Leucht S, Komossa K, Rummel-Kluge C, et al. A meta-analysis of head-to-head comparisons of second-generation antipsychotics in the treatment of schizophrenia. Am J Psychiatry 2009; 166: 152-163.

[3] Rummel-Kluge C, Komossa K, Schwarz S, et al. Head-to-head comparisons of metabolic side effects of second generation antipsychotics in the treatment of schizophrenia: A systematic review and meta-analysis. Schizophr Res 2010; 123: 225-233.

[4] Rummel-Kluge C, Komossa K, Schwarz S, et al. Second-generation antipsychotic drugs and extrapyramidal side effects: A systematic review and meta-analysis of head-to-head comparisons. Schizophr Bull 2012; 38: 167-177.

[5] Hasan A, Falkai P, Wobrock T, et al. World Federation of Societies of biological Psychiatry (WFSBP) guidelines for biological treatment of schizophrenia, Part 2: update 2012 on the long-term treatment of schizophrenia and management of antipsychotic-induced side effects. World J Biol Psychiatry 2013; 14: 2-44.

[6] Hasan A, Falkai P, Wobrock T, et al. World Federation of societies of biological psychiatry (WFSBP) guidelines for biological treatment of schizophrenia, Part 1: update 2012 on the acute treatment of schizophrenia and the management of treatment resistance. World J Biol Psychiatry 2012; 13: 318-378.

[7] Lehman AF, Lieberman JA, Dixon LB, et al. Practice guideline for the treatment of patients with schizophrenia, second edition. Am J Psychiatry. 2004; 161: 1-56.

[8] Shapiro DA, Renock S, Arrington E, et al. Aripiprazole, a novel atypical antipsychotic drug with a unique and robust pharmacology. Neuropsychopharmacology 2003; 28: 14001411.

[9] Stark AD, Jordan S, Allers KA, et al. Interaction of the novel antipsychotic aripiprazole with 5-HT1A and 5-HT 2A receptors: Functional receptor-binding and in vivo electrophysiological studies. Psychopharmacology (Berl) 2007; 190: 373-382.

[10] Leucht S, Cipriani A, Spineli L, et al. Comparative efficacy and tolerability of 15 antipsychotic drugs in schizophrenia: A multipletreatments meta-analysis. Lancet 2013; 382: 951-962.

[11] Buchanan RW, Kreyenbuhl J, Kelly DL, et al. 
The 2009 schizophrenia PORT psychopharmacological treatment recommendations and summary statements. Schizophr Bull 2010; 36: 7193.

[12] Kuipers E, Yesufu-Udechuku A, Taylor C, et al. Management of psychosis and schizophrenia in adults: Summary of updated NICE guidance. BMJ 2014; 348: g1173.

[13] Noda Y, Kurumiya S, Miura Y, et al. Comparative study of 2-(4-ethyl-1-piperazinyl)-4-(fluorophenyl )-5,6,7,8,9,10-hexahydrocycloocta [b ] pyridine (AD-5423) and haloperidol for their pharmacological activities related to antipsychotic efficacy and/or adverse side-effects. J Pharmacol Exp Ther 1993; 265: 745-751.

[14] Oka M, Noda Y, Ochi Y, et al. Pharmacological profile of AD-5423, a novel antipsychotic with both potent dopamine-D2 and serotoninS2 antagonist properties. J Pharmacol Exp Ther 1993; 264: 158-165.

[15] Kishi T, Matsuda Y, Nakamura H, et al. Blonanserin for schizophrenia: Systematic review and meta-analysis of double-blind, randomized, controlled trials. J Psychiatr Res 2013; 47: 149-154.

[16] Hori H, Yamada K, Kamada D, et al. Effect of blonanserin on cognitive and social function in acute phase Japanese schizophrenia compared with risperidone. Neuropsychiatr Dis Treat
2014; 10: 527-533.

[17] Kishi T, Matsuda Y, Matsunaga S, et al. A randomized trial of aripiprazole vs blonanserin for the treatment of acute schizophrenia and related disorders. Neuropsychiatr Dis Treat 2016; 12: 3041-3049.

[18] Inada T. Evaluation and diagnosis of druginduced extrapyramidal symptoms: Commentary of the DIEPSS and guide to its usage. Seiwa Shoten, Tokyo, 1996.

[19] Hamilton M. The assessment of anxiety states by rating. Br J Med Psychol 1959; 32: 50-55.

[20] Montgomery SA, Asberg M. A new depression scale designed to be sensitive to change. The British Journal of Psychiatry 1979; 134: 382389.

[21] Buckley PF, Correll CU. Strategies for dosing and switching antipsychotics for optimal clinical management. J Clin Psychiatry 2008; 69; Suppl 1: 4-17.

[22] Correll CU. From receptor pharmacology to improved outcomes: Individualising the selection, dosing, and switching of antipsychotics. Eur Psychiatry 2010; 25; Suppl 2: S12-S21.

[23] Takeuchi H, Kantor N, Uchida H, et al. Immediate vs Gradual Discontinuation in Antipsychotic Switching: A Systematic Review and Meta-analysis. Schizophr Bull 2017; 43: 862871. 